\title{
Performance improvement of pentacene based organic field-effect transistor with HfON gate insulator
}

\author{
Min Liao $^{1 \mathrm{a})}$, Hiroshi Ishiwara ${ }^{1,2}$, and Shun-ichiro Ohmi ${ }^{1 \mathrm{~b}}$ ) \\ ${ }^{1}$ Department of Electronics and Applied Physics, Tokyo Institute of Technology, \\ J2-72, 4259 Nagatsuta, Midori-ku, Yokohama 226-8502, Japan \\ 2 Department of Physics, Division of Quantum Phases and Devices, \\ Konkuk University, Seoul 143-701, Korea \\ a) liao.m.aa@m.titech.ac.jp \\ b)ohmi@ep.titech.ac.jp
}

\begin{abstract}
Pentacene based organic field-effect transistors (OFETs) with $\mathrm{SiO}_{2}$ and HfON gate insulators have been fabricated, and the effect of deposition temperature and deposition rate on the grain sizes of pentacene films was investigated. It was found that the grain sizes of pentacene films increase with increasing deposition temperature and decreasing deposition rate. Due to the increase in grain size, pentacene based OFET with HfON gate insulator shows enhanced electrical properties, such as a low subthreshold swing of $0.14 \mathrm{~V} /$ decade and a large on/off current ratio of $1.1 \times 10^{4}$. Moreover, the hole mobility of pentacene based OFET with HfON gate insulator is $0.39 \mathrm{~cm}^{2} / \mathrm{Vs}$ at an operating voltage of $-2 \mathrm{~V}$.
\end{abstract}

Keywords: pentacene, OFETs, HfON, grain size, electrical properties

Classification: Electronic materials, semiconductor materials

\section{References}

[1] C. D. Dimitrakopoulos and P. R. L. Malenfant, "Organic thin film transistors for large area electronics," Adv. Mater., vol. 14, no. 2, pp. 99-117, Jan. 2002.

[2] H. Klauk, "Organic circuits on flexible substrates," IEDM Technical Digest, Washington, America, pp. 446-449, Dec. 2005.

[3] P. T. Liu, Y. T. Chou, and Y. Y. Kao, "Improvement of electron-gun evaporated aluminum oxide for pentacene thin-film transistor," Electrochem. Solid-State Lett., vol. 12, no. 1, pp. H11-H13, Jan. 2009.

[4] S. Tatemichi, M. Ichikawa, S. Kato, T. Koyama, and Y. Taniguchi, "Low-voltage, high-gain, and high-mobility organic complementary inverters based on N,N'-ditridecyl-3,4,9,10-perylenetetracarboxylic diimide and pentacene," Phys. Stat. Sol. (RRL), vol. 2, no. 2, pp. 47-49, March 2008.

[5] J. M. Choi, D. K. Hwang, S. H. Jeong, J. H. Park, E. Kim, J. H. Kim, and $\mathrm{S}$. Im, "Polymer $/ \mathrm{AlO}_{\mathrm{x}}$ bilayer dielectrics for low-voltage organic thin-film 
transistors," J. Electrochemical Society, vol. 154, no. 5, pp. H331-H335, May 2007.

[6] A. R. Völkel, R. A. Street, and D. Knipp, "Carrier transport and density of state distributions in pentacene transistors," Phys. Rev. B, vol. 66, no. 19, pp. 195336(1-8), Nov. 2002.

[7] F. J. M. Heringdorf, M. C. Reuter, and R. M. Tromp, "Growth dynamics of pentacene thin films," Nature, vol. 412, no. 6846, pp. 517-520, Aug. 2001.

[8] C. L. Fan, T. H. Yang, P. C. Chiu, C. H. Huang, and C. I. Lin, "Organic thin-film transistor performance improvement using ammonia $\left(\mathrm{NH}_{3}\right)$ plasma treatment on the gate insulator surface," Solid-State Electronics, vol. 53, no. 2, pp. 246-250, Feb. 2009.

[9] Y. Y. Lin, D. J. Gundlach, S. F. Nelson, and T. N. Jackson, "Stacked pentacene layer organic thin-film transistors with improved characteristics," IEEE Electron Device Lett., vol. 18, no. 12, pp. 606-608, Dec. 1997.

[10] L. Shang, M. Liu, D. Tu, G. Liu, X. Liu, and Z. Ji, "Low-voltage organic field-effect transistor with PMMA/ZrO $\mathrm{Z}_{2}$ bilayer dielectric," IEEE Trans. Electron Devices, vol. 56, no. 3, pp. 370-376, March 2009.

[11] C. B. Park, K. D. Jung, S. H. Jin, B. G. Park, and J. D. Lee, "Pentacenebased thin film transistors with improved mobility characteristics using hybrid gate insulator," J. Information Display, vol. 6, no. 2, pp. 16-18, June 2005.

[12] M. Liao, H. Ishiwara, and S. Ohmi, "Effect of gate insulator on the electrical properties of pentacene based organic field-effect transistors," IEICE Technical Report, vol. 110, no. 241, pp. 49-52, Oct. 2010.

[13] T. Sano and S. Ohmi, " $\mathrm{HfO}_{\mathrm{x}} \mathrm{N}_{\mathrm{y}}$ thin-film formation on three-dimensional Si structure utilizing electron cyclotron resonance sputtering," Jpn. J. Appl. Phys., vol. 48, no. 5, pp. 05DB04(1-4), May 2009.

[14] S. Ohmi and Y. Nakano, "Investigation of PDA process to improve electrical characteristics of $\mathrm{HfO}_{\mathrm{x}} \mathrm{N}_{\mathrm{y}}$ high-k dielectric formed by ECR plasma oxidation of HfN," IEEE Int. Symp. Semiconductor Manufacturing, California, America, pp. 514-517, Oct. 2007.

[15] L. S. Tsai, C. H. Wang, W. Y. Chen, W. C. Wang, and J. Hwang, "Lowvoltage organic thin-film transistors with hydrophobic hafnium oxynitride film as gate insulator," Organic Electronics, vol. 11, no. 1, pp. 123126, Jan. 2010.

\section{Introduction}

Organic field-effect transistors (OFETs) have attracted considerable interest in the electronics industry due to their potential advantages such as lightweight, high flexibility, low cost, and high volume throughput compared to conventional Si devices [1]. Pentacene is one of the most widely investigated organic semiconductors for OFETs because of its high intrinsic hole mobility [2]. But the operating voltages required to achieve high hole mobility $(\mu)$ from pentacene based OFETs are generally higher than $20 \mathrm{~V}$. In order to address this issue, high-k gate insulators were employed in the pentacene based OFETs [3, 4]. However, high-k gate insulator usually has a large surface energy [5], which results in small grain size for pentacene films. Due to 
the small pentacene grain size, the $\mu$ of pentacene based OFET with high-k gate insulator is generally low. In addition to the carrier mobility, subthreshold swing $(S S)$ and on/off current ratio $\left(I_{o n} / I_{\text {off }}\right)$ must also be optimized to ensure proper operation of the OFETs. However, trap states are usually formed by air exposure and fabrication process at grain boundaries and the gate insulator interface $[6,7,8,9]$, which will decrease $I_{o n} / I_{o f f}$ and increase $S S$ of organic devices. Although some reports revealed that large $I_{\text {on }} / I_{\text {off }}$ can be obtained by using hybrid gate insulators, such as $\mathrm{PMMA} / \mathrm{ZrO}_{2}$ and PMMA/PVA/SiO 2 , the $S S$ values for these kinds of OFETs are high [10, 11], which would result in low switching speed when used in logic circuit.

In this paper, we reported that the grain sizes of pentacene films increase with increasing deposition temperature and decreasing deposition rate. Due to the increase in grain size, pentacene based OFET with HfON gate insulator exhibits enhanced electrical properties, such as a low $S S$ of $0.14 \mathrm{~V} /$ decade, a large $I_{\text {on }} / I_{\text {off }}$ of $1.1 \times 10^{4}$ and a high $\mu$ of $0.39 \mathrm{~cm}^{2} / \mathrm{Vs}$ at an operating voltage of $-2 \mathrm{~V}$.

\section{Experimental procedure}

In our investigation, pentacene based OFETs were fabricated on $\mathrm{n}^{+}-\mathrm{Si}(100)$ substrates with a top-contact bottom-gate device geometry [12]. HfON gate insulators were fabricated on $\mathrm{n}^{+}-\mathrm{Si}(100)$ substrates by electron cyclotron resonance (ECR) plasma sputtering [13]. Firstly, $\mathrm{n}^{+}-\mathrm{Si}(100)$ substrates were chemically cleaned followed by the formation of chemical oxide layers, and the thickness of chemical oxide layer is $0.7 \mathrm{~nm}$. Then, HfN thin films $(5 \mathrm{~nm})$ were deposited on the substrates by ECR plasma sputtering at room temperature, and the gas pressure in the chamber during the deposition was $0.19 \mathrm{~Pa}\left(\mathrm{Ar} / \mathrm{N}_{2}\right.$ flow rate: $\left.25 / 1 \mathrm{sccm}\right)$. In order to form HfON films, the HfN films were oxidized by ECR Ar $/ \mathrm{O}_{2}$ plasma irradiation, and the gas pressure for oxidation was $0.18 \mathrm{~Pa}\left(\mathrm{Ar} / \mathrm{O}_{2}\right.$ flow rate: $\left.20 / 8 \mathrm{sccm}\right)$. Finally, HfON films were annealed by silicon-wafer-covering rapid thermal annealing (SWC-RTA) method at $800^{\circ} \mathrm{C}$ for 1 minute in $\mathrm{N}_{2}$ ambient [14]. The final thickness of HfON gate insulator was $9.5 \mathrm{~nm}$.

For comparison, $\mathrm{SiO}_{2}(9.5 \mathrm{~nm})$ films were grown on $\mathrm{n}^{+}-\mathrm{Si}(100)$ substrates by wet thermal oxidation at $850^{\circ} \mathrm{C}$. After the fabrication of gate insulators, pentacene films (from Aldrich without any purification) were deposited on gate insulators by thermal evaporation at room temperature and $70^{\circ} \mathrm{C}$, respectively. The pressure in the chamber was $2 \times 10^{-6}$ Torr. The deposition rate was changed from 0.1 to $0.01 \mathrm{~nm} / \mathrm{s}$. The thickness of the pentacene films was $20 \mathrm{~nm}$. After the pentacene deposition, Au electrodes were evaporated through a shadow mask to form source and drain electrodes for OFETs. Channel width $(W)$ and length $(L)$ of the OFETs were 1400 and $50 \mu \mathrm{m}$, respectively. Atomic force microscopy (AFM) was employed to characterize the morphologies of pentacene films, and the electrical properties of the OFETs were evaluated by semiconductor parameter analyzer (Agilent 4156C). And the measurements were carried out in air. 


\section{Results and discussion}

Figures 1 (a) and 1(b) show the AFM images of pentacene films deposited on $\mathrm{SiO}_{2}$ and $\mathrm{HfON}$ gate insulators at a deposition temperature of $70^{\circ} \mathrm{C}$ and a deposition rate of $0.01 \mathrm{~nm} / \mathrm{s}$. Clearly, the grain size for pentacene film deposited on HfON gate insulator is much larger than that for pentacene film deposited on $\mathrm{SiO}_{2}$ gate insulator. The average grain sizes for pentacene films deposited on $\mathrm{SiO}_{2}$ and $\mathrm{HfON}$ gate insulators at this condition are 0.9 and $2.5 \mu \mathrm{m}$, respectively.

The average grain sizes of pentacene films deposited on $\mathrm{SiO}_{2}$ and $\mathrm{HfON}$ gate insulators as a function of deposition temperature and deposition rate are summarized in Figs. 2 (a) and 2(b). It is found that the grain sizes of pentacene films increase with decreasing the deposition rate when the deposition temperature is kept at room temperature. The grain sizes of pentacene films further increase when the deposition temperature increases to $70^{\circ} \mathrm{C}$. And pentacene films deposited at a deposition temperature of $70^{\circ} \mathrm{C}$ and a deposition rate of $0.01 \mathrm{~nm} / \mathrm{s}$ show the largest grain size. However, the increment in grain size for pentacene film deposited on HfON gate insulator
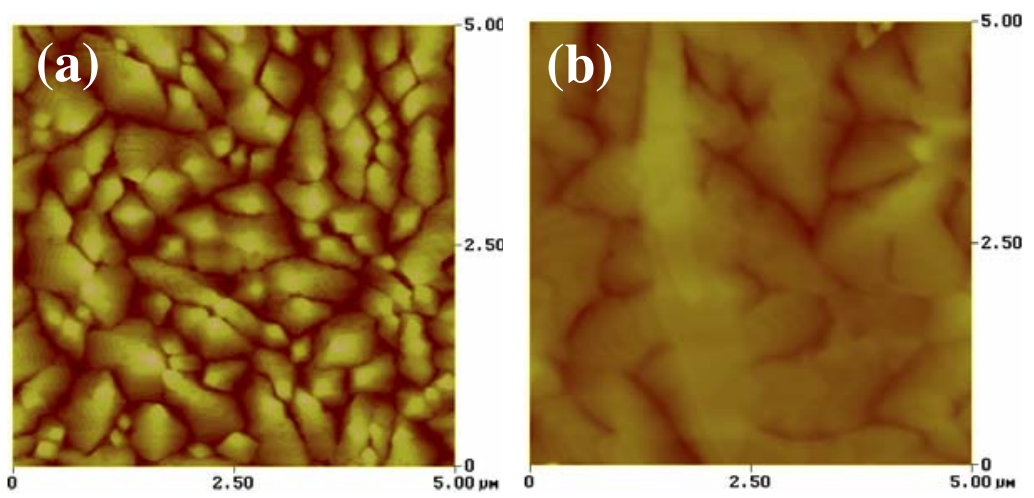

Fig. 1. AFM images of pentacene films deposited on (a) $\mathrm{SiO}_{2}$ and (b) HfON gate insulators at a deposition temperature of $70^{\circ} \mathrm{C}$ and a deposition rate of $0.01 \mathrm{~nm} / \mathrm{s}$. The scanning size is $5 \times 5 \mu \mathrm{m}$.
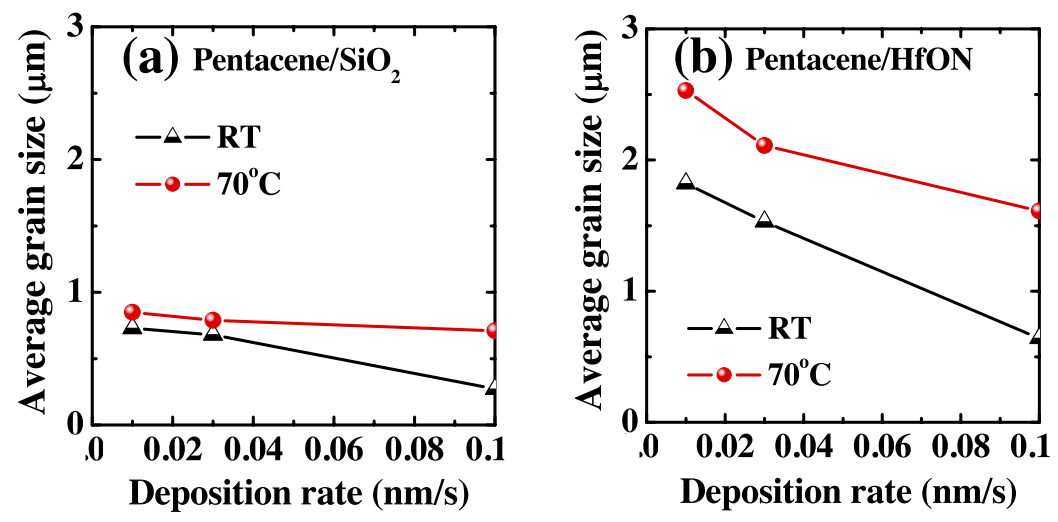

Fig. 2. Average grain sizes of pentacene films deposited on (a) $\mathrm{SiO}_{2}$ and (b) HfON gate insulators as a function of deposition temperature and deposition rate. 

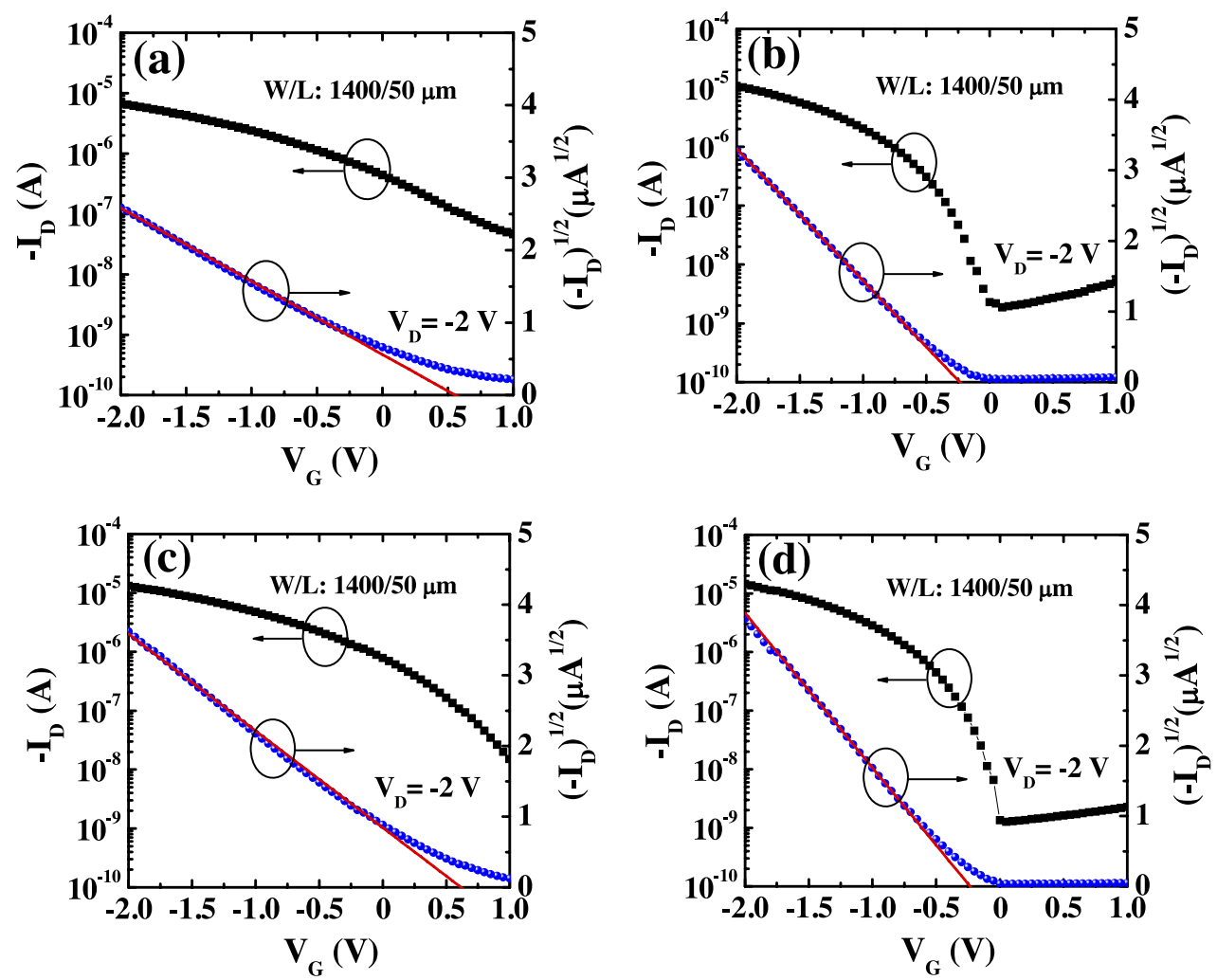

Fig. 3. $I_{D}-V_{G}$ characteristics of pentacene based OFETs with (a) $\mathrm{SiO}_{2}$ and (b) HfON gate insulators, in which pentacene films were deposited at room temperature and a deposition rate of $0.1 \mathrm{~nm} / \mathrm{s}$. $\mathrm{I}_{\mathrm{D}}-\mathrm{V}_{\mathrm{G}}$ characteristics of pentacene based OFETs with (c) $\mathrm{SiO}_{2}$ and (d) HfON gate insulators, in which pentacene films were deposited at a deposition temperature of $70^{\circ} \mathrm{C}$ and a deposition rate of $0.01 \mathrm{~nm} / \mathrm{s}$.

is more remarkable than that for pentacene film deposited on $\mathrm{SiO}_{2}$ gate insulator. It is attributed to the lower surface energy of HfON compared to $\mathrm{SiO}_{2}[5,15]$.

Figure 3 shows the $I_{D}-V_{G}$ characteristics of pentacene based OFETs with $\mathrm{SiO}_{2}$ and HfON gate insulators, in which pentacene films were deposited at different conditions. The off current $\left(I_{\text {off }}\right)$ for OFETs in this paper is defined as the drain current at zero gate bias. As shown in Fig. 3, the fabricated OFETs can successfully operate at a low operating voltage of $-2 \mathrm{~V}$. However, the magnitude of $I_{\text {off }}$ shows a dependence on the gate insulator, and pentacene based OFET with HfON gate insulator shows a lower $I_{\text {off }}$ compared to pentacene based OFET with $\mathrm{SiO}_{2}$ gate insulator. For pentacene films deposited at room temperature and a deposition rate of $0.1 \mathrm{~nm} / \mathrm{s}$, the $\mu, I_{\text {on }} / I_{\text {off }}$ and $S S$ of OFET with $\mathrm{SiO}_{2}$ gate insulator are $0.16 \mathrm{~cm}^{2} / \mathrm{Vs}, 15$ and $0.93 \mathrm{~V} /$ decade, respectively. The performance of the pentacene based OFET can be enhanced by using HfON gate insulator, which leads to a $\mu$ of $0.19 \mathrm{~cm}^{2} / \mathrm{Vs}$, an $I_{\text {on }} / I_{\text {off }}$ of $5.0 \times 10^{3}$ and a $S S$ of $0.18 \mathrm{~V} /$ decade. From Fig. 3, the electrical properties of the pentacene based OFETs are found to 
be enhanced by increasing deposition temperature and decreasing deposition rate. For pentacene films deposited at a deposition temperature of $70^{\circ} \mathrm{C}$ and a deposition rate of $0.01 \mathrm{~nm} / \mathrm{s}$, OFET with $\mathrm{SiO}_{2}$ gate insulator shows a $\mu$ of $0.25 \mathrm{~cm}^{2} / \mathrm{Vs}$, an $I_{\text {on }} / I_{\text {off }}$ of 17 and a $S S$ of $0.44 \mathrm{~V} /$ decade, respectively. The $\mu$ and $I_{\text {on }} / I_{\text {off }}$ of pentacene based OFET with HfON gate insulator increases to $0.39 \mathrm{~cm}^{2} / \mathrm{Vs}$ and $1.1 \times 10^{4}$, respectively, while the $S S$ of the OFET decreases to $0.14 \mathrm{~V} /$ decade.

As shown in Figs. 2 (a) and 2 (b), the average grain sizes for pentacene films deposited on $\mathrm{SiO}_{2}$ and $\mathrm{HfON}$ gate insulators at room temperature and a deposition rate of $0.1 \mathrm{~nm} / \mathrm{s}$ are 0.3 and $0.6 \mu \mathrm{m}$, respectively. It is known that the grain boundaries in pentacene films can act as trapping sites [6], and such trapping sites may lead to low $\mu$, high $S S$ and small $I_{\text {on }} / I_{\text {off }}[7,8,9]$. On the other hand, the average grain sizes for pentacene films deposited on $\mathrm{SiO}_{2}$ and $\mathrm{HfON}$ gate insulators at a deposition temperature of $70^{\circ} \mathrm{C}$ and a deposition rate of $0.01 \mathrm{~nm} / \mathrm{s}$ are 0.9 and $2.5 \mu \mathrm{m}$, respectively. Due to the increased grain sizes of pentacene films, the grain boundaries which act carrier traps are reduced. Therefore, the electrical properties of pentacene based OFETs are improved greatly by increasing deposition temperature and decreasing deposition rate. However, because of the remarkable increase in pentacene grain size, pentacene based OFET with HfON gate insulator shows excellent electrical properties, such as a lower $S S$ of $0.14 \mathrm{~V} /$ decade, a larger $I_{\text {on }} / I_{\text {off }}$ of $1.1 \times 10^{4}$ and a higher $\mu$ of $0.39 \mathrm{~cm}^{2} / \mathrm{Vs}$ at an operating voltage of $-2 \mathrm{~V}$. Meanwhile, due to the larger pentacene grain size, the hole mobility of pentacene based OFET with HfON gate insulator in this paper is higher than the results $\left(0.17 \mathrm{~cm}^{2} / \mathrm{Vs}\right)$ reported by Tsai et al. [15].

\section{Conclusion}

In conclusion, pentacene based OFETs with $\mathrm{SiO}_{2}$ and $\mathrm{HfON}$ gate insulators have been fabricated, and the effect of deposition parameters on the grain sizes of pentacene films was investigated. Our results show that the grain sizes of pentacene films increase with increasing deposition temperature and decreasing deposition rate. However, the grain size for pentacene film deposited on HfON gate insulator is larger than that for pentacene film deposited on $\mathrm{SiO}_{2}$ gate insulator. The large grain size of pentacene film should play an important role in the good electrical properties of pentacene based OFET with HfON gate insulator.

\section{Acknowledgments}

This work was supported by the WCU (World Class University) program through the NRF funded by Ministry of Education, Science and Technology, Korea (no. R31-2008-000-10057-0). A part of this work was supported by a Grant-in-Aid for JSPS Fellows under Grant 237982. The authors would like to thank Dr. Y. Fujisaki of Hitachi, Drs. M. Shimada and K. Saito of MES-Afty, and Dr. Y. Jin of NTT for their support and useful discussions. 